\title{
unciación Enunelacion \\ Por el camino de la evaluación institucional en la Universidad Distrital Francisco José de Caldas
}

\author{
By the way of the institutional \\ assessment in the Universidad \\ Distrital Francisco José de Caldas
}

\section{Resumen}

El presente documento sintetiza una reflexión de los diversos aportes y experiencias surgidas de la adopción del proceso de autoevaluación en la Universidad Distrital Francisco José de Caldas. La experiencia se desarrolla alrededor de dos estrategias principales: la autoevaluación/autorregulación y la participación. Ello permite que la vivencia pueda leerse desde la propuesta de impulsar una cultura de la calidad, en la que la autoevaluación se constituye en un mecanismo importante para el logro de los objetivos institucionales, y la acreditación en un medio para rendir cuentas a la sociedad.

\footnotetext{
1 Licenciada en Educación con énfasis en Matemáticas, Universidad Distrital Francisco Jose de Caldas, Bogota.Magíster en Desarrollo Educativo y Social. Universidad Pedagógica Nacional CINDE. Bogota. Correo electrónico: acreditacion@udistrital.edu.co

2 Licenciada en Ciencias Sociales. Universidad Externado de Colombia, Bogota Magíster en Ciencia Política, Universidad de los Andes, Bogota. Correo electrónico: acreditacion@udistrital.edu.co
} 


\section{Palabras clave: evaluación institucional, autoevaluación, autorregulación, acreditación.}

\begin{abstract} way to accountability to society.

\section{Institutional Assessment, Self Assessment, Self-Regulation, Accreditation.}

The following document summarizes a reflection of the diverse experiences and contributions arisen from the adoption of the self-evaluation process at the Distrital University Francisco José de Caldas. The process was developed around two main strategies: self-evaluation / self-regulation and the participation. This allows the experience can be read from the proposal to promote the quality culture, in which the self-evaluation process is a vital mechanism in order to achieve the institutional objectives and the accreditation in a

\section{Introducción}

El tema de la evaluación de instituciones y de programas curriculares no es nuevo; es más bien un tema recurrente, por lo menos, desde las políticas educativas. En las últimas dos décadas ingresó a la realidad universitaria latinoamericana, a través de la implementación de las políticas educativas nacionales integradas a los sistemas de aseguramiento de la calidad (Brunner, 1993; Orozco, 2006), concebida como un instrumento ligado a dos propósitos: garantizar el mejoramiento de la calidad de la educación superior y promover la rendición de cuentas a la sociedad por parte de estas instituciones. En Colombia este sistema, creado en la Ley 30 de 1992 y desarrollado por las políticas del Ministerio de Educación Nacional (MEN) subsiguientes, incluye procesos internos de evaluación, realizados por la instituciones de educación superior, procesos externos promovidos por el gobierno nacional a través del Ministerio de Educación Nacio- nal y las acciones de las agencias de aseguramiento de la calidad (Consejo Nacional de Acreditación [CNA] y Comisión Intersectorial para la Calidad de la Educación Superior [Conaces]). El conjunto de instrumentos de política creados incluye la evaluación de programas y de instituciones, así como la aplicación de evaluaciones masivas sobre el aprendizaje de los estudiantes y la adopción de sistemas de información únicos y de obligatorio sostenimiento.

La discusión sobre la conveniencia de incluir dichas políticas en la agenda de las universidades latinoamericanas, y en particular de las universidades públicas colombianas, ha tenido un recorrido salpicado de críticas acerca de su intencionalidad dado el origen de la propuesta.

\section{Marco de referencia}

La evaluación académica de las instituciones de educación superior (Brunner, 1990) surge en los organismos de crédito internacional, es impuesta a los gobiernos con el ánimo de hacer que la educación superior sea funcional a los intereses del modelo de economía neoliberal (Orozco, 2006; Vargas, 2002) y fue operativizada a través de las políticas establecidas en cada país. La evaluación académica surge en América Latina como una expresión del establecimiento de nuevas relaciones entre Universidad y Estado, pues tal como lo afirma (Brunner, 1993), tales políticas son el resultado del desmonte del "Estado benevolente", hacia el establecimiento del "Estado evaluador":

El surgimiento del Estado evaluador puede interpretarse como el principal efecto del mencionado reacomodo en las relaciones entre gobiernos e instituciones de educación superior. En todas partes parece responder al deseo de racionalizar esas relaciones en base a un control a distancia de las instituciones, librándolas de regulaciones burocráticas minuciosas y exponiéndolas, por el contrario, a las señales que dan los mercados (Brunner, 1993, p. 99). 
El establecimiento de estos nuevos discursos se argumenta, por parte del Estado colombiano, a partir del reconocimiento de la crisis de la calidad de la educación y de la necesidad de que las instituciones educativas rindan cuentas a la sociedad, fundamentándose en una mirada de la calidad que enfatiza la eficiencia, la eficacia y la productividad, para desde allí deducir que la mencionada crisis no obedece a la falta de recursos sino a una crisis gerencial y de gestión.

Las críticas que algunos sectores académicos han realizado a estas políticas y al establecimiento de estas nuevas relaciones Estado-Universidad, se asientan en la defensa de la autonomía universitaria y en la consideración de que los resultados obtenidos tendrán su correlato en la financiación que el Estado hará a las universidades, específicamente a las públicas. Consideran que evaluación y financiación son los mecanismos a través de los cuales el Estado establece la nueva regulación y el control hacia las universidades, incentivando los procesos de autorregulación que deben derivar en procesos de cambio y mejoramiento, con lo cual se impulsa la homogenización de la educación, para dar respuesta a las demandas de la globalización y a los intereses de los mercados trasnacionales (Vargas, 2002). Se señala entonces que la constitución y consolidación de los mecanismos de evaluación académica no tienen en cuenta aspectos propios de las realidades que afectan a las diversas sociedades, realidades contextualizadas en las historias del modelo educativo y de desarrollo económico y social que cada país ha recorrido.

A pesar de las críticas, consideradas por algunos como acertadas, los sistemas de aseguramiento de la calidad se han asentado en los países y han cobrado carta de ciudadanía, teniendo como propósitos generales ser garantes de la calidad de la educación impartida por las instituciones de educación superior (IES) (Orozco, 2010). De tal suerte que las instituciones de educación superior en Colombia, poco a poco, se han visto incorporadas en un cúmulo de demandas impuestas a través del MEN denominadas por Orozco (2010) "turbulencias", que analizadas de manera global indican contraposiciones entre lo que es la Universidad tradicional y lo que los grupos de interés presionan para que ella sea: una universidad funcional al mercado:

Para muy pocos de estos grupos cuentan los valores básicos que defienden y practican algunas universidades consolidadas: la libertad intelectual, la autonomía, el valor de la crítica y el interés de la universidad en la formación integral de sus egresados. Por el contrario, a la universidad se le pide que produzca conocimiento de frontera sin profesores bien formados; que capacite profesionales de manera rápida y poco costosa, y que se articule a la solución de os problemas del país, sin que las propuestas de quienes detentan el poder puedan ser discutidas. Los grupos de interés requieren una "universidad funcional"orientada por los mercados (Chaparro, 2007; Clark, 1997) (Orozco, 2010, p.29).

Rechazando la visión de la educación superior como mercancía inmersa en las leyes del mercado, académicos nacionales e internacionales reclaman las reformas y el establecimiento de los sistemas de aseguramiento de la calidad de la educación superior, partiendo, como lo hace Dias Sobrinho (2008), de caracterizarla como:

[...] bien público, derecho de todos, deber del Estado. Esta premisa afirma dos cosas importantes: a) no solo todos tienen derecho a la educación, como también es un deber del Estado proveer a todos una educación de calidad; b) la educación no es un bien negociable, aunque pueda ser impartida también por actores privados. Al ser pública, la educación tiene que ser de calidad para todos. Por ello, son beneficiosos los mecanismos que cumplan con las funciones de promover y asegurar la calidad de las instituciones de educación superior (IES) de acuerdo con las demandas prioritarias de la sociedad. En tanto bien público, el aseguramiento de la calidad no puede transformarse en un interesante rubro de comercio, sino que es imprescindible que sea un instrumento de profundización de los valores democráticos, fortalecimiento de la soberanía nacional y la identidad nacional (Dias Sobrinho, 2008, p.87)

Bajo estas condiciones, sin que los análisis y las reflexiones acerca de la evaluación y la acreditación hayan terminado, se estableció en la Ley 30 de 1992 el interés por fomentar la calidad de la educación, otorgando al Estado la función de regular y ejercer la suprema inspección y vigilancia de la educación, con el fin de velar por su calidad en consonancia con el mandato de la Constitución de 1991, la cual señala la educación como un derecho de la persona y un servicio público con función social. Con estos referentes, en el país se inicia desde 1994 el proceso de fundamentación de 
todo el sistema de aseguramiento de la calidad de la educación, en el marco del cual la universidad Colombiana y particularmente la Universidad Distrital Francisco José de Caldas, diseñan e implementan sistemas de calidad que son elevados a nivel de política y por tanto son introducidos en sus planes de desarrollo.

Así, como lo afirma Hoyos (s.f.) la acreditación debe concebirse como un medio que permita la creación de nuevas relaciones Universidad-Estado y sociedad civil, en las que la práctica comunicativa, participativa, tenga un lugar privilegiado:

Para ello los procesos de acreditación deben crear un clima en el que sea posible verificar la calidad como el conjunto de acciones en las que interactúan, por un lado, normas, políticas, propuestas, es tándares y resultados, de alguna manera evaluables y controlable inclusive por parte del Estado; por otro, comunidades científicas en constante comunicación interna y externa; y finalmente las ex pectativas de la sociedad civil, urgida de cambios e innovaciones pertinentes (p. 17)

\section{La autoevaluación y la acreditación en la Universidad Distrital Francisco José de Caldas}

La Universidad Distrital Francisco José de Caldas, desde hace más de diez años (1997), asumió los compromisos de mejoramiento de la calidad y desarrollo de la autoevaluación y la acreditación como una de sus políticas, particularmente en 1999. La Vicerrectoría Académica de esa época, presentó la propuesta denominada Modelo de Evaluación Institucional para la Universidad Distrital Francisco José de Caldas, el cual fue propuesto por el grupo de investigación evaluativa universitaria. Esta propuesta se incorporó al Plan de Desarrollo "Por la Universidad que queremos"; en dicho modelo se propuso hacer de la evaluación y la autoevaluación prácticas sociales y culturales, que tienen como propósito indagar e intervenir sobre la realidad institucional con el fin de alcanzar transformaciones (Lozano et ál., 1999).

Los planteamientos anteriores se han venido ratificando en el Proyecto Institucional (PUI), en el Plan de Desarrollo "Educación de Calidad para equidad social, 2001-2005" y en el Plan de Desarrollo 20072016 "Saberes, Conocimientos e Investigación de Alto
Impacto para el Desarrollo Humano y Social". En el transcurso de esta década, de manera paulatina, los esfuerzos de la Universidad se han centrado en que su quehacer académico y administrativo esté orientado hacia la búsqueda de mejores niveles de calidad, diseñando para ello dos estrategias complementarias: la autoevaluación/autorregulación y la participación. La acreditación se ha considerado un ejercicio de rendición de cuentas que será el resultado del establecimiento de la cultura de la autoevaluación permanente establecida en la práctica participativa.

Así, el proceso de autoevaluación se conceptualiza como un cambio cultural que implica "el crecimiento de la conciencia individual y colectiva sobre el sentido de nuestras acciones y la valoración sobre la calidad social de las acciones de investigación, docencia, extensión y acción cultural universitaria, [...] que nos permitirá estudiar y estudiarnos, investigar e investigarnos, descubrir el potencial y la multiplicidad de singularidades y generalidades, diversidades y pluralidades de la comunidad universitaria" (Universidad Distrital Francisco José de Caldas, 2002, p. 58), estableciendo como uno de los resultados de la autoevaluación la construcción de un proceso de control permanente, que ejercido por la misma institución, autorregulación, se constituye en el vigilante del cumplimiento de los objetivos institucionales acordados en el ejercicio de la planeación participativa.

Una consecuencia de asumir tal postura, es considerar la evaluación institucional como una "oportunidad de participación directa de la comunidad universitaria en la generación de espacios de reflexión, orientados al estudio de textos y contextos educativos y culturales, el reconocimiento de logros y proyectos de transformación en las concepciones, teorías, métodos, condiciones, procesos y prácticas universitarias" (Universidad Distrital Francisco José de Caldas, 2002, p. 59).

Un paso inicial para el desarrollo de la política de autoevaluación lo constituyó la discusión y adopción de 
Por el camino de la evaluación institucional en la Universidad Distrital Francisco José de Caldas

un concepto propio de calidad. Así, se asumió la calidad como un concepto histórico-contextual, dinámico, multidimensional y multirreferencial, ligado a los conceptos de desarrollo humano y ciudadano, pertinencia y equidad, y enmarcado en el cumplimiento de sus propios propósitos institucionales.

En este sentido, el desarrollo de la política de calidad en la Universidad Distrital Francisco José de Caldas implica que se reconozca como universidad pública que realiza su quehacer académico con el propósito de lograr la democratización del acceso al conocimiento para garantizar el derecho a una educación superior rigurosa, crítica, de calidad y con condiciones de equidad, y en procura de crear, recrear y recuperar conocimientos y saberes socialmente pertinentes $y$ formar profesionales competentes y comprometidos con el desarrollo de la ciencia, la tecnología, el arte y la cultura para el desarrollo humano y la transformación sociocultural de la nación colombiana y la ciudad región, reafirmando la responsabilidad social como expresión de la autonomía.

De tal manera que el concepto de calidad de la educación superior para la Universidad Distrital Francisco José de Caldas está ligado a un enfoque de garantía de derechos, incorporando las dimensiones de equidad, pertinencia, responsabilidad social, eficiencia y eficacia. Todo ello concretado en nuestro lema "Autoevaluación y Acreditación: Compromiso Social".

\section{Un balance de nuestra experiencia}

El enfoque de la calidad ligado a la garantía de un derecho, concretado en la democratización del acceso al conocimiento y el compromiso con el desarrollo humano y la transformación sociocultural de la ciudad región y el país en el marco de la internacionalización y la globalización, permite a todos los proyectos curriculares evaluar sus propuestas de formación en torno a estos enunciados y definir su misión asumiendo su responsabilidad en la formación integral de ciudadanos, provenientes de los sectores más vulnerables y marginados de la sociedad, en especial del Distrito Capital. Así, hemos comprendido y afianzado el sentido social de nuestra acción educativa, en tanto tenemos como norte, para la transformación y el mejoramiento, el deber de brindar una educación con calidad y pertinencia, que le ofrezca a todos los estudiantes las mismas oportunidades en el ingreso, la permanencia y la graduación, en el marco del reconocimiento de sus diferencias, para que alcancen niveles de desarrollo y aprendizajes que les permita ser ciudadanos activos, profesionales altamente competentes y seres humanos proclives al respeto por los derechos humanos y al fortalecimiento de la democracia.

Además de consolidar cada vez más nuestra identidad y posibilidad institucional, la evaluación institucional se ha incrustado como una más de las actividades que resultan importantes, pertinentes y necesarias de realizar, tarea que ha resultado posible aunque llena de altibajos.

Proponerse cambios culturales obtenidos en medio de procesos participativos resulta ser una empresa lenta y muchas veces difícil, pero no imposible. Ese es nuestro caso. Haremos un breve recorrido por algunos de los momentos en que estas dos estrategias: cambio cultural y participación, resultaron exitosas y por lo tanto permitieron avances en nuestras indagaciones y conocimientos de nosotros mismos.

Uno de los hitos fundamentales lo constituyó la creación del Comité Institucional de Autoevaluación y Acreditación, cuya estructura se construye considerando la participación de la comunidad académica, ya que sus integrantes son profesores y estudiantes adscritos a las cinco facultades en las que se organiza la universidad y a los diversos proyectos curriculares de pregrado y posgrado. Recientemente se ha creado el Comité Técnico de Autoevaluación y Acreditación Institucional que junto con el Sistema Integrado de 
Calidad (Sigud), conformarán la estructura orgánica responsable de los procesos de calidad, transformación institucional y sostenibilidad.

Otro hito, de gran importancia académica, lo constituyó la adopción de un modelo de autoevaluación que definió los referentes, la estructura, implementación, los instrumentos, la metodología, los planes de mejoramiento y su seguimiento. Estos elementos constituyeron la carta de navegación que utilizaron todos los proyectos curriculares para realizar las correspondientes autoevaluaciones y en el caso en que se consideró oportuno presentarse a los procesos de acreditación. De este primer proceso se obtuvo un resultado de 18 proyectos curriculares acreditados de alta calidad, con vigencias entre tres y siete años. En un segundo proceso se han recibido nuevas acreditaciones y hemos incursionado, con buenos resultados, en los procesos de reacreditación, que tienen como característica importante el que se demuestra nuestro compromiso con el mejoramiento continuo.

Dada la continuidad del proceso y su condición de proyecto en permanente construcción, constitución y desarrollo, un nuevo reto institucional se concreta en el cumplimiento con los compromisos de mejoramiento y transformación, por lo que un hito importante lo constituyó la utilización de los resultados de la autoevaluación en los procesos de planeación institucional y su concreción en los planes de desarrollo, en particular del plan del 2007-2016, uno de cuyos insumos principales corresponde precisamente a la necesidad de que la Universidad de respuesta a los resultados de la investigación evaluativa (autoevaluación), respetando las diversidades y pluralidades propias de una institución productora de conocimiento.

En medio de estos procesos relacionados con la autoevaluación se implementan otras estrategias de evaluación, como es el caso de la evaluación a la labor docente, en las que participan los profesores, los estudiantes y los consejos curriculares. Esta evaluación, que se realiza en línea, es ahora respondida por casi el 70\% de los estudiantes, la totalidad de los profesores y todos los consejos curriculares. Sus resultados son utilizados para estimular a los profesores por el buen desempeño, así como para detectar necesidades de formación, sobre todo pedagógicas, de los profesores que obtienen bajos niveles de calificación. Este aspecto es importante, en tanto convencer al cuerpo docente de una institución universitaria pública, de que su desempeño es posible de evaluar al tiempo que sus resultados le pueden servir para transformar algunas de sus prácticas, es un cambio cultural difícil de lograr, pero que hoy es reconocido por toda la comunidad académica.

Así las cosas, hoy podemos afirmar que aunque ha sido un proceso lento, los resultados obtenidos nos permiten mostrar que en la Universidad se ha ganado en pertinencia, a la vez que se ha consolidado el carácter de institución pública que rinde cuentas a la sociedad, como un ejercicio de la autonomía y autorregulación.

La discusión sobre la calidad y los resultados de la autoevaluación han permitido que las propuestas de reformas o adecuaciones curriculares incorporen dimensiones sobre la pertinencia, la equidad y la calidad de la oferta educativa. Como consecuencia de ello, se han realizado actividades que requieren la participación de los profesores, los estudiantes, los directivos y los administrativos, lo que redunda en mayor conocimiento y pertenencia con el proyecto curricular y sus propuestas de mejoramiento.

El reconocimiento de la utilidad de los sistemas de información no sólo ha incrementado la presión institucional porque la Universidad tenga un sistema de información unificado y eficiente, sino que ha permeado a todos aquellos que producen o validan información. Ello trae consigo que la Universidad ratifique su apuesta por lo público y por la gestión transparente y eficiente. 
Por el camino de la evaluación institucional en la Universidad Distrital Francisco José de Caldas

Por último, la presencia de pares externos que han mirado nuestra institución, y particularmente sus proyectos curriculares, ha estimulado el debate y la cooperación académica, así como ha mejorado la mirada interna sobre lo que somos y podemos ser. Exponerse a otros implica conocerse a sí mismo, de tal modo que hoy podemos asegurar que a la vez que hemos ganado en conocimiento interno, intensificamos la interacción con el medio externo, en el marco de procesos de comunicación académica.

En síntesis, los procesos de evaluación han permeado la institución a tal punto que hoy podemos afirmar que la actividad evaluativa ha ingresado a la cotidianidad institucional, tanto en el ámbito de las funciones sustantivas como de la administración de la universidad.

\section{Los riesgos del proceso}

Como todo proceso en el que intervienen seres humanos, en este también aparecen riesgos asociados, de los cuales destacamos la posibilidad de convertir el proceso en burocrático en tanto se asuma como una tarea a realizar, como el cumplimiento de procesos externos regulados por el Estado, lo que consideramos puede suceder entre otras cuestiones ya que:

1. Se aumenta el volumen de trabajo de los profesores responsables del proceso, quienes empiezan a sentir que estas labores caen fuera de sus intereses investigativos o de trabajo en extensión.

2. La interacción proyecto curricular-mejoramiento y compromiso de las directivas de la institución, requerida para que los procesos fluyan, puede resultar una de las trabas fundamentales para que existan procesos de transformación y compromiso real.

3. La falta de una política de seguimiento y control, en particular las emanadas del Consejo Nacional de Acreditación, puede llevar a las instituciones y sus programas a que se asuma el proceso como discreto y no como continuo.

4. La posibilidad de que los resultados se generen pensando en lo que los pares desearían oír, y no como un proceso de autorreconocimiento y autorregulación, se ve incrementada por la subjetividad de algunos pares al realizar los informes, lo que perjudica enormemente el proceso de reconocimiento institucional y de incremento del sentido de autonomía y pertenencia institucional.

5. Se asuman los procesos para mejorar lo existente y no para innovar y transformar.

6. La participación sin conocimiento sobre los procesos y sobre los propósitos institucionales es lesiva para el desarrollo institucional, puesto que induce a que los debates se orienten por las concepciones de sentido común o los intereses individuales sin que medie la institucionalidad y su racionalidad.

7. La participación como proceso de comunicación y debate puede hacer lentos los procesos, por ello se requiere que haya una planeación sobre el sentido y los alcances de esta.

\section{Conclusiones}

Concebir la política de autoevaluación/autorregulación como un proceso eminentemente participativo y a la vez como un proceso en el que el mejoramiento y la innovación son sus resultados más importantes, ha permitido a la Universidad Distrital Francisco José de Caldas iniciar procesos de cambio cultural, que esperamos continúen para transformar la Universidad en una universidad investigativa como se afirma en nuestro escenario apuesta:

Sentar las bases que posibiliten el desarrollo de la Institución hacia la consolidación de las condiciones "necesarias y medios adecuados para proyectarse como una universidad investigativa de alto impacto en la solución de problemas de la ciudad-región de Bogotá y el país en la formación de profesionales integrales en las diversas áreas del conocimiento, comprometidos con los procesos socioculturales de su contexto" $y$, fortalecer $y$ articular las funciones misionales de docencia, investigación y proyección social de manera integrada, con énfasis en la consolidación de las condiciones que permitan proyectar su acreditación institucional, académica y social. (Plan decenal de desarrollo 2007 - 2016, p 87) 


\section{Reconocimiento}

Esta reflexión es producto de los proceso de autoevaluación bajo la coordinación de la Oficina de Autoevaluación y Acreditación Institucional de la Universidad Distrital Francisco José de Caldas.

\section{Referencias}

Brunner J. J. (1990). Bases para un nuevo contrato entre el Estado y la educación superior. Documento de trabajo. Santiago de Chile.

- (1993). Algunos criterios para la evaluación de la educación superior y la investigación. Notas para el debate No. 8. Lima: Grade.

- (2003) Aseguramiento de la calidad y nuevas demandas sobre la educación superior en América Latina. En CNA, Educación superior, calidad y acreditación, (vol. I). Bogotá: Consejo Nacional de Acreditación.

Consejo Nacional de Acreditación (2003). Educación Superior, Calidad y Acreditación. Tomo I.

Dias Sobrinho, J. (2008a). Calidad, pertinencia y responsabilidad social de la universidad latinoamericana y Caribeña. En Tendencias de la Educación Superior en América Latina. CRES

- (2008b). Cambios y Reformas en la Educación Superior. En Tünnermann Bernheim, C. (Ed.), La educación superior en América Latina y el Caribe: diez años después de la Conferencia Mundial de 1998. Cali: lesalc-Unesco, PUJ.
Hoyos, G. (s.f.). Participación del estado, de la comunidad académica y de la sociedad en el mejoramiento de la calidad de la educación superior. Bogotá: Consejo Nacional de Acreditación.

Lozano, F. et ál. (1999). Propuesta de modelo de evaluación institucional para la Universidad Distrital. Bogotá: Sección de publicaciones Universidad Distrital Francisco José de Caldas.

Orozco, L. E. (2003). La Calidad de las Universidades. Más allá de toda ambigüedad. Bogotá: Consejo Nacional de Acreditación.

- (2006). Tareas pendientes de las universidades en América Latina. Quórum, Revista de pensamiento latinoamericano, (015): 77-92.

- (2010). Calidad académica y relevancia social de la educación superior en América Latina. Revista Iberoamericana de Educación Superior (RIES), 1(1). Recuperado de http://ries.universia.net.mx/index.php/ ries/article/view/22/calidad_academica

Universidad Distrital Francisco José de Caldas (2002). Lineamientos de autoevaluación y acreditación institucional. Bogotá: Sección de publicaciones Universidad Distrital Francisco José de Caldas.

(2005). Proyecto Universitario Institucional “Educación de calidad para equidad social, 2001-2005".

(2007). Plan de Desarrollo 2007-2016 "Saberes, conocimientos e investigación de alto impacto para el desarrollo humano y social".

Vargas, A. (2002). La acreditación: una forma de estandarizar la educación. Educación, 26(002): 245-254. 